\title{
VARIABILIDADE ESPACIAL DE CARACTERÍSTICAS FÍSICO-HÍDRICAS E DE ÁGUA DISPONÍVEL EM UM SOLO ALUVIAL NO SEMI-ÁRIDO PARAIBANO ${ }^{1}$
}

\author{
João Rodrigues de Sousa² ${ }^{2}$ José Elenildo Queiroz $^{3}$ \& Hans Raj Gheyi ${ }^{4}$
}

\begin{abstract}
RESUMO
Este trabalho teve como objetivo avaliar a variabilidade espacial de características físico-hídricas e de água disponível de um solo aluvial para fins de manejo adequado da irrigação. Amostras de solo com estrutura deformada foram coletadas (profundidades de 0 - 0,20 e 0,20 - 0,40 m) numa área do Projeto Capoeira, Município de São José do Bonfim, Estado da Paraíba, através de uma amostragem sistemática (malha regular de $10 \times 15 \mathrm{~m}$ ), totalizando 90 pontos; foi realizada uma análise descritiva geral e identificada a estrutura espacial de variância das seguintes variáveis: porcentagens de areia, silte e argila, capacidade de campo (CC), ponto de murcha permanente (PMP) e porcentagem de água disponível (AD). Constatou-se variabilidade média para as variáveis estudadas, com CV entre 17,43\% (\% argila de 0,20 - 0,40 m) e 46,48\% (\% areia de 0,20 - 0,40 m). Com base na amplitude interquartílica (Ai) observou-se a seguinte ordem decrescente de variabilidade: $\%$ areia $>\%$ silte $>\%$ argila $>\mathrm{CC}>\mathrm{AD}>$ PMP; observou-se dependência espacial, com alcances variando de $25 \mathrm{~m}$ (\% silte de $0-0,20 \mathrm{~m}$ ) a $60 \mathrm{~m}$ (\% silte de 0,20 - 0,40 m). O mapa de isolinhas para AD, construído com valores estimados mediante o processo de krigagem, permitiu uma visualização do seu padrão de variabilidade, constituindo uma importante ferramenta para orientação do manejo da irrigação.
\end{abstract}

Palavras-chave: variabilidade, água disponível, dependência espacial, geoestatística

\section{SPATIAL VARIABILITY OF HYDRO-PHYSICAL CHARACTERISTCS AND AVAILABLE WATER IN ALLUVIAL SOIL IN THE SEMI-ARID REGION OF PARAÍBA STATE}

\footnotetext{
ABSTRACT

The aim of the present work was to evaluate the spatial variability of hydro-physical characteristics and available water of an alluvial soil, keeping in view adequate irrigation management. Disturbed soil samples ( 0 - 0.20 and 0.20 - 0.40 m layers) were collected in the Capoeira Project, São José do Bonfim,

${ }^{1}$ Parte do trabalho de dissertação de mestrado apresentado ao Curso de Pós-Graduação em Engenharia Agrícola da Universidade Federal da Paraíba, Campus II

${ }^{2}$ Eng. Agrônomo, M.Sc. em Engenharia Agrícola, Prof. Substituto da CEFET-PB, Rua José Antônio da Silva S/N, Jardim Oásis, CEP 59900 - 000, Cajazeiras, PB. Telefax: (083) 531 4560, Ramal 214

${ }^{3}$ Prof. Adjunto do Departamento de Engenharia Florestal da UFPB/CSTR/Campus VII, CP 64, CEP 58700 - 970 , Patos, PB. E-mail: elenildo@cstr.ufpb.br

${ }^{4}$ Prof. Titular do Departamento de Engenharia Agrícola da UFPB/CCT-Campus II. Av. Aprígio Veloso 882, CEP 58109 - 970 Campina Grande, PB. Fone: (083) 310 1056, Fax: (083) 310 1185, E-mail:hans@ deag.ufpb.br
} 
Paraíba state, Brazil. Systematic scheme was used on the regular grid $(10$ x $15 \mathrm{~m})$ in all 90 points. Descriptive analyses were performed to identify spatial structure of the following variables: sand, silt, clay, field capacity (FC), permanent wilting point (PWP) and percent available water(AW). Mean variability was found for studied variables, with coefficient of variation (CV) between $17.43 \%$ (clay, 0.20 - $0.40 \mathrm{~m}$ layer) and $46.48 \%$ (sand, $0.20-0.40 \mathrm{~m}$ layer). The quarter interval showed decreasing sequence of variability: sand $>$ silt $>$ clay $>$ FC $>$ AW $>$ PWP. The studied variables presented spatial dependence, with range varying between $25 \mathrm{~m}$ (silt, 0 - $0.20 \mathrm{~m}$ layer) and $60 \mathrm{~m}$ (silt, $0.20-0.40 \mathrm{~m}$ layer). The isohypsas map, constructed with estimated AW value by kriging, was observed to be an important tool for the irrigation management.

Key words: variability, available water, spatial dependence, geostatistics

\section{INTRODUÇÃO}

O solo é um corpo natural complexo e dinâmico, resultante da atuação conjunta de muitos fatores de formação, como clima, organismos vivos, material de origem, relevo e tempo. Dependendo da intensidade de atuação ou da predominância de alguns desses fatores, são formados solos de diferentes características químicas e físicas. Durante a pedogênese pode haver adição, remoção, transformação e translocação de elementos químicos do material original (Oliveira, 1972; Vieira et al., 1988). Esses processos determinam, em maior ou menor intensidade, as características químicas e físicas dos solos, conferindo-lhes grande variabilidade, dependendo de onde estão situados. Beckett \& Webster (1971) indicam as diferenças climáticas regionais como um dos fatores responsáveis pela variação dos solos e acrescentam, ainda, a ação do homem como fator de variabilidade, principalmente nas características químicas.

Os solos aluviais são ainda mais problemáticos, podendo exibir diferenças significativas nas suas propriedades e características em pequenas dimensões, tanto vertical como horizontalmente, acarretando problemas de manejo de solo e água. Esses solos, apesar dos problemas típicos, são muito utilizados para a agricultura, o que justifica a necessidade de pesquisas que propiciem melhor compreensão de sua variabilidade espacial, para aprimoramento das técnicas de manejo de solo e água.

Nesse contexto, o conhecimento da variabilidade espacial da capacidade de retenção de água e de outras características correlacionadas com a disponibilidade de água do solo, é de fundamental importância no manejo da irrigação, permitindo o zoneamento da área em glebas que receberão manejo diferenciado.

O presente trabalho teve por objetivo avaliar a variabilidade espacial de variáveis físico-hídricas de um solo aluvial no semi-árido paraibano, utilizando-se métodos da estatística descritiva e procedimentos geoestatísticos para identificação da magnitude de variabilidade e da dependência espacial das variáveis analisadas, com o fim de se proceder ao mapeamento da água disponível na área estudada e fornecer subsídios para um manejo mais adequado da irrigação.

\section{MATERIAL E MÉTODOS}

O estudo foi conduzido em solo aluvial do Projeto Capoeira, localizado na microbacia do Rio da Cruz (conhecido como Rio Capoeira), parte da bacia do Rio Piancó (localizada entre os

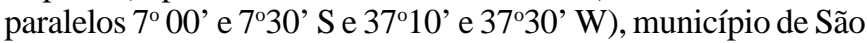
José do Bonfim, Estado da Paraíba. Segundo a classificação de Köppen, o clima da região é Bsh-semi-árido quente e seco e a precipitação média é de $400 \mathrm{~mm}$ com estação seca muito acentuada. As temperaturas médias nunca são inferiores a $24^{\circ} \mathrm{C}$ com amplitude de $5^{\circ} \mathrm{C}$ (SUDENE, 1972). Os solos são do tipo aluvial, representados pelas unidades Ae1, Ae2, Ae3 e Ae4 (Governo do Estado da Paraíba, 1989).

Foi realizada uma amostragem sistemática em malha regular de $10 \times 15 \mathrm{~m}$ (90 pontos) em duas profundidades ( 0 - 0,20 e 0,20 - 0,40 m) totalizando 180 amostras. As seguintes variáveis físico-hídricas foram analisadas: retenção de água a $33 \mathrm{kPa}$ (capacidade de campo-CC) e a $1500 \mathrm{kPa}$ (ponto de murcha permanente - PMP) e textura, conforme EMBRAPA (1997). A água disponível foi obtida por diferença entre CC e PMP e expressa em porcentagem.

Procedeu-se a uma análise estatística descritiva geral, para verificação do comportamento dos dados quanto às medidas estatísticas de posição e de variabilidade, bem como a aderência à distribuição normal. Nessas análises, assume-se que as observações são independentes espacialmente, isto é, as variações de um lugar para outro são consideradas aleatórias. Seu objetivo não é fazer qualquer tipo de inferência, mas analisar o comportamento inicial dos dados.

A dependência espacial foi avaliada por meio de recursos geoestatísticos, utilizando-se o semivariograma construído a partir da estimativa das semivariâncias pela expressão:

$$
\hat{\gamma}(h)=\frac{1}{2 N(h)} \sum_{i=1}^{N(h)}\left[Z\left(x_{i}\right)-Z\left(x_{i}+h\right)\right]^{2}
$$

em que $\hat{\gamma}(h)$ é o valor estimado da semivariância dos dados experimentais, $\mathrm{Z}\left(\mathrm{x}_{\mathrm{i}}\right)$ e $\mathrm{Z}\left(\mathrm{x}_{\mathrm{i}}+\mathrm{h}\right)$ são os valores observados da variável regionalizada e $\mathrm{N}(\mathrm{h})$ é o número de pares de valores medidos, separados por uma distância h (Guerra, 1988).

Os semivariogramas experimentais foram escalonados através da divisão das semivariâncias pela variância estatística s² (Guimarães, 1993; Vieira et al., 1997). Com as semivariâncias adimensionalizadas, o efeito pepita expressa diretamente, em porcentagem do patamar (semivariância total), o componente do acaso da estrutura de variância. De acordo com Guimarães (1993) verifica-se proporcionalidade quando os semivariogramas escalonados permitem o ajuste de um único modelo para a variável em estudo. Neste trabalho os semivariogramas foram escalonados com o objetivo de reduzi-los à mesma escala, facilitando a comparação entre resultados de diferentes variáveis.

Aos semivariogramas experimentais escalonados ajustou-se um modelo tipo esférico, conforme as Eqs. (2) e (3):

$$
\begin{array}{cc}
\gamma(\mathrm{h})=\mathrm{Co}+\mathrm{Cl}\left[\frac{3}{2}\left(\frac{\mathrm{h}}{\mathrm{a}}\right)-\frac{1}{2}\left(\frac{\mathrm{h}}{\mathrm{a}}\right)^{3}\right] ; & 0<\mathrm{h} \leq<\mathrm{a} \\
\gamma(h)=C o+C 1 ; & \mathrm{h} \geq \mathrm{a}
\end{array}
$$

onde Co é o efeito pepita, $\mathrm{Co}+\mathrm{C} 1$ é o patamar, hé a distância de separação entre duas observações e a é o alcance de dependência espacial. 


\section{RESULTADOS E DISCUSSÃO}

$\mathrm{Na}$ Tabela 1 constam as estatísticas para os componentes texturais areia, silte e argila, expressos em porcentagem. Os valores médios obtidos permitem classificar o solo como francoargiloso (Kiehl, 1979). Pelos valores dos coeficientes de variação (CV) pode-se constatar a seguinte ordem de variabilidade: areia > silte > argila. Os valores de CV entre 17,43 e 46,48\%, permitem classificar os resultados como de média variabilidade, conforme Warrick \& Nielsen (1980).

Tabela 1. Resumo estatístico das porcentagens de areia, silte e argila

\begin{tabular}{|c|c|c|c|c|c|c|}
\hline \multirow[t]{3}{*}{ Estatísticas } & \multicolumn{2}{|c|}{ Areia $(\%)$} & \multicolumn{2}{|c|}{ Silte $(\%)$} & \multicolumn{2}{|c|}{ Argila (\%) } \\
\hline & \multicolumn{6}{|c|}{$\overline{\text { Profundidade }(\mathrm{m})}$} \\
\hline & $0-0,20$ & $0,20-0,40$ & $0-0,20$ & $0,20-0,40$ & $0-0,20$ & $0,20-0,40$ \\
\hline Média & 34,43 & 30,53 & 32,58 & 34,53 & 32,99 & 34,95 \\
\hline Mediana & 35,03 & 29,35 & 32,92 & 34,67 & 33,61 & 35,28 \\
\hline Variância $\left(S^{2}\right)$ & 221,93 & 201,32 & 124,34 & 102,49 & 39,01 & 37,09 \\
\hline Desvio-padrão (S) & 14,90 & 14,19 & 11,15 & 10,12 & 6,25 & 6,09 \\
\hline $\mathrm{CV}(\%)$ & 43,27 & 46,48 & 34,23 & 29,32 & 18,93 & 17,43 \\
\hline Curtose (Cr) & 2,70 & 2,40 & 2,47 & 2,24 & 2,91 & 2,66 \\
\hline Assimetria (Cs) & 0,40 & 0,51 & $-0,15$ & $-0,12$ & 0,09 & $-0,16$ \\
\hline Amplitude total (At) & 68,39 & 58,37 & 48,84 & 43,96 & 32,73 & 26,91 \\
\hline Menor & 9,85 & 8,74 & 4,04 & 11,86 & 17,72 & 21,03 \\
\hline Maior & 78,24 & 67,11 & 52,87 & 55,82 & 50,45 & 47,94 \\
\hline Amp. Interquartílica (Ai) & 22,26 & 21,95 & 16,48 & 15,36 & 9,01 & 8,23 \\
\hline Primeiro quartil & 23,31 & 17,05 & 23,93 & 27,50 & 28,26 & 30,66 \\
\hline Terceiro quartil & 45,57 & 39,00 & 40,41 & 42,85 & 37,26 & 38,90 \\
\hline
\end{tabular}

A proximidade de valores entre média e mediana e os valores de assimetria e curtose próximos de $0 \mathrm{e} 3$, respectivamente sugerem distribuição normal para os dados (Spiegel, 1985). Entretanto, o teste de Kolmogorv-Smirnov (KS) a nível de 5\% de significância, conforme Sousa (1998), só confirma esta hipótese para argila $(0-0,20$ e $0,20-0,40 \mathrm{~m})$, silte $(0-0,20 \mathrm{~m})$ e areia $(0,20-0,40 \mathrm{~m})$.

Os valores de CC e PMP, obtidos a base de massa e expressos em porcentagem (Tabela 2), possuem magnitudes de variabilidade semelhantes. A variável $\mathrm{AD}$, apesar de derivada das anteriores, possui CV ligeiramente maior, o que está de acordo com Moraes \& Libardi (1993) ao afirmarem que nem sempre uma variável obtida

Tabela 2. Resumo estatístico da retenção de água a 33 e 1500 $\mathrm{kPa}$ e porcentagem de água disponível

\begin{tabular}{|c|c|c|c|c|c|c|}
\hline \multirow{4}{*}{ Estatísticas } & \multicolumn{4}{|c|}{ Retenção de Água } & \multirow{2}{*}{\multicolumn{2}{|c|}{$\frac{\text { Água Disponível }}{(\% \mathrm{AD})}$}} \\
\hline & \multicolumn{2}{|c|}{$33 \mathrm{kPa}(\mathrm{CC})$} & \multicolumn{2}{|c|}{$1500 \mathrm{kPa}(\mathrm{PMP})$} & & \\
\hline & \multicolumn{6}{|c|}{ Profundidade $(\mathrm{m})$} \\
\hline & $0-0,20$ & $0,20-0,40$ & $0-0,20$ & $0,20-0,40$ & $0-0,20$ & $0,20-0,40$ \\
\hline Média & 20,71 & 21,85 & 10,43 & 11,04 & 10,28 & 10,81 \\
\hline Mediana & 20,57 & 21,88 & 10,19 & 11,08 & 10,31 & 10,67 \\
\hline Variância $\left(\mathrm{S}^{2}\right)$ & 28,26 & 27,46 & 7,59 & 6,59 & 8,18 & 9,38 \\
\hline Desvio-padrão (S) & 5,32 & 5,24 & 2,76 & 2,57 & 2,86 & 3,06 \\
\hline $\mathrm{CV}(\%)$ & 25,67 & 23,98 & 26,42 & 23,26 & 27,82 & 28,34 \\
\hline Curtose (Cr) & 2,77 & 2,92 & 2,78 & 2,62 & 2,61 & 3,53 \\
\hline Assimetria (Cs) & 0,12 & 0,02 & 0,14 & $-0,22$ & 0,04 & 0,53 \\
\hline Amplitude total (At) & 25,17 & 27,14 & 13,35 & 11,94 & 13,89 & 15,47 \\
\hline Menor & 7,76 & 9,80 & 3,95 & 4,60 & 3,81 & 4,92 \\
\hline Maior & 32,93 & 36,94 & 17,30 & 16,54 & 17,70 & 20,40 \\
\hline Amp. interquartílica (Ai) & 7,13 & 7,14 & 3,46 & 4,01 & 4,36 & 3,95 \\
\hline Primeiro quartil & 16,91 & 18,50 & 8,83 & 9,19 & 8,09 & 8,59 \\
\hline Terceiro quartil & 24,04 & 25,64 & 12,29 & 13,21 & 12,46 & 12,53 \\
\hline
\end{tabular}

de forma algébrica possui o mesmo comportamento das variáveis que lhe deram origem. Quanto à normalidade, apenas CC $(0,20-0,40 \mathrm{~m})$ satisfez esta hipótese, conforme o teste de KS a nível de 5\% de significância (Sousa, 1998).

A análise exploratória dos dados realizada por Souza (1998), através da construção de gráficos "box-plot" nas direções X e Y da malha experimental demonstrou ser razoável a aceitação da hipótese da estacionaridade, constatando um comportamento semelhante dos componentes texturais e das variáveis de retenção de água ( CC, PMP e AD ). As análises mostraram que as variáveis estudadas exibiram variação na direção $X$ da malha experimental sem um tendência capaz de comprometer esta hipótese.

Todas as variáveis estudadas apresentaram estrutura de dependência espacial, conforme os semivariogramas escalonados e seus respectivos modelos teóricos ajustados (Figuras 1 e 2 e Tabela 3).

O alcance de dependência espacial é um dos parâmetros importantes do semivariograma e define a distância máxima até onde o valor de uma variável possui relação de dependência com o seu próximo (Guerra, 1988). O menor e o maior alcances de dependência espacial verificaram-se para porcentagem de silte nas profundidades de $0-0,20$ e 0,20 - 0,40 m (25 e $60 \mathrm{~m}$, respectivamente).

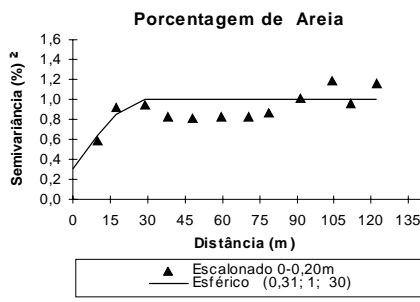

A

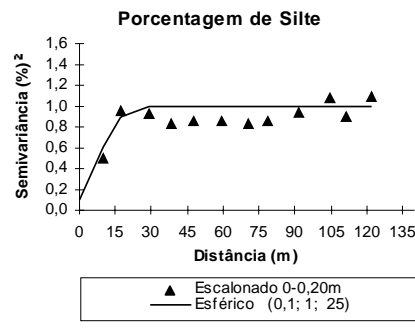

$\mathrm{C}$

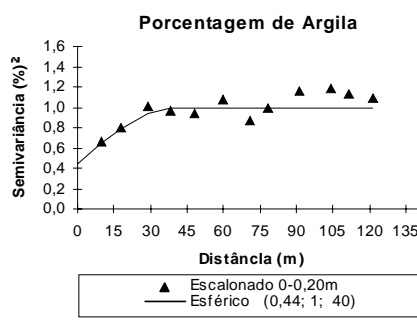

E

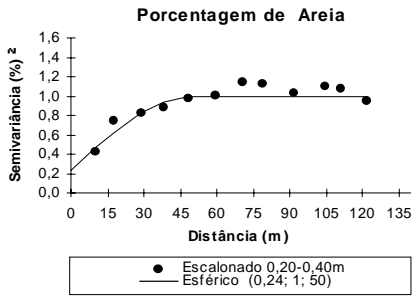

B

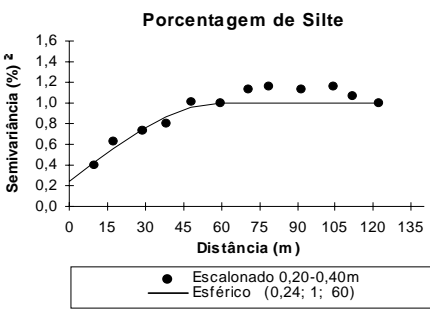

D

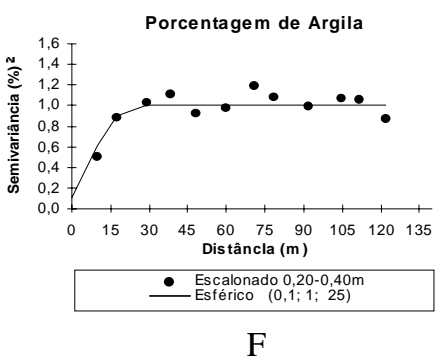

Figura 1. Semivariograma escalonado e modelo esférico ajustado para porcentagem de areia (A, B), silte (C, D) e argila (E, F) nas profundidades de $0-0,20 \mathrm{~m} \mathrm{e} 0,20-0,40 \mathrm{~m}$

$\mathrm{O}$ efeito pepita reflete a variabilidade não explicada ou variações não detectadas pela distância insuficiente da malha de amostragem (Cambardella et al., 1994). Quando expresso como porcentagem da semivariância total (patamar), possibilita melhor 


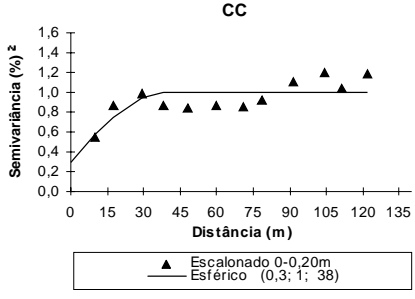

A

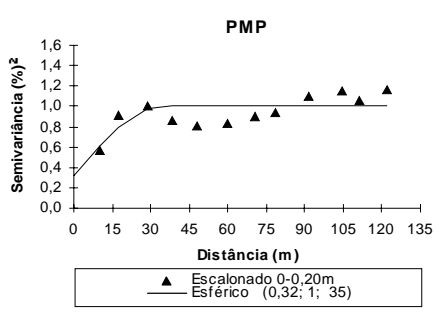

$\mathrm{C}$

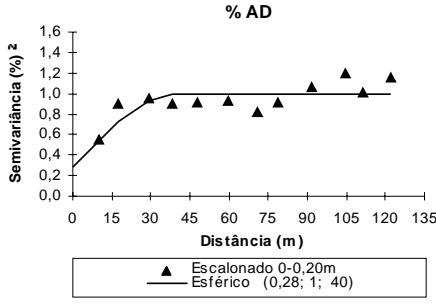

$\mathrm{E}$

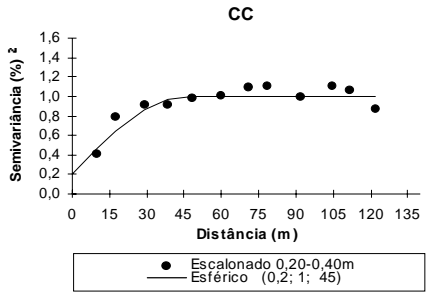

B

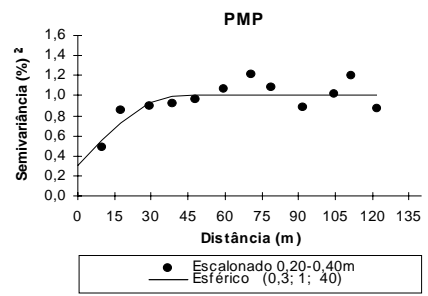

D

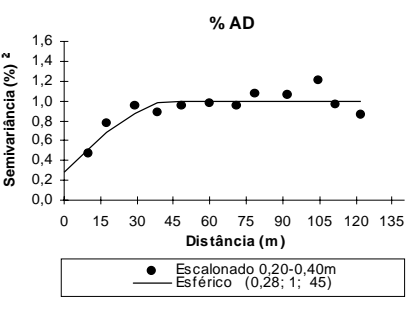

$\mathrm{F}$
Figura 2. Semivariograma escalonado e modelo esférico ajustado para CC (A, B), PMP (C, D) e AD (E, F) nas profundidades de $0-0,20 \mathrm{me} 0,20-0,40 \mathrm{~m}$

comparação entre as variáveis quanto ao grau de dependência espacial (Trangmar et al., 1985). De acordo com os critérios propostos por Cambardella et al. (1994) semivariogramas com efeito pepita $<25 \%$, entre 25 e $75 \%$ e $>75 \%$, são considerados como de forte, moderada e fraca dependência espacial, respectivamente.

Na Tabela 3 o efeito pepita, expresso como uma porcentagem do patamar $[\mathrm{Co} /(\mathrm{Co}+\mathrm{C} 1)] .100$ mostra que na profundidade de $0-0,20 \mathrm{~m}$, apenas a porcentagem de silte possui forte dependência espacial, sendo as demais classificadas como de moderada dependência espacial; na profundidade de 0,20 - $0,40 \mathrm{~m}$, observase forte dependência espacial das variáveis texturais e da CC, e uma dependência espacial moderada no caso da PMP e AD. Com exceção de silte houve um predomínio de dependência espacial moderada na camada superficial, o que pode ser resultado do manejo do solo. Salviano (1996) verificou que

Tabela 3. Parâmetros do modelo esférico ajustado aos semivariogramas experimentais escalonados e efeito pepita, expresso em porcentagem

\begin{tabular}{|c|c|c|c|c|c|c|c|}
\hline \multicolumn{2}{|r|}{ Co } & \multicolumn{2}{|c|}{$\mathrm{Co}+\mathrm{C} 1$} & \multicolumn{2}{|r|}{$\mathrm{a}(\mathrm{m})$} & \multicolumn{2}{|c|}{$[\mathrm{Co} /(\mathrm{Co}+\mathrm{C} 1)] .100$} \\
\hline \multicolumn{8}{|c|}{ Profundidade ( $\mathrm{m}$ ) } \\
\hline $0-0,20$ & $0,20-0,40$ & $\overline{0-0,20}$ & $0,20-0,40$ & $0-0,20$ & $0,20-0,40$ & $\overline{0-0,20}$ & $0,20-0,40$ \\
\hline 0,31 & 0,24 & 1,0 & 1,0 & 30 & 50 & 31 & 24 \\
\hline 0,10 & 0,24 & 1,0 & 1,0 & 25 & 60 & 10 & 24 \\
\hline 0,44 & 0,10 & 1,0 & 1,0 & 40 & 25 & 44 & 10 \\
\hline 0,30 & 0,20 & 1,0 & 1,0 & 38 & 45 & 30 & 20 \\
\hline 0,32 & 0,30 & 1,0 & 1,0 & 35 & 40 & 32 & 30 \\
\hline 0,28 & 0,28 & 1,0 & 1,0 & 40 & 45 & 28 & 28 \\
\hline
\end{tabular}

variações extrínsecas, devido ao manejo do solo, causaram menores valores de dependência espacial na superfície do mesmo.

Devido à similaridade de comportamento observado para as semivariâncias de $\mathrm{AD}$ nas duas profundidades estudadas (Figuras 2E e F) decidiu-se construir um semivariograma único com as semivariâncias das médias de $\mathrm{AD}$, conforme a Figura 3.

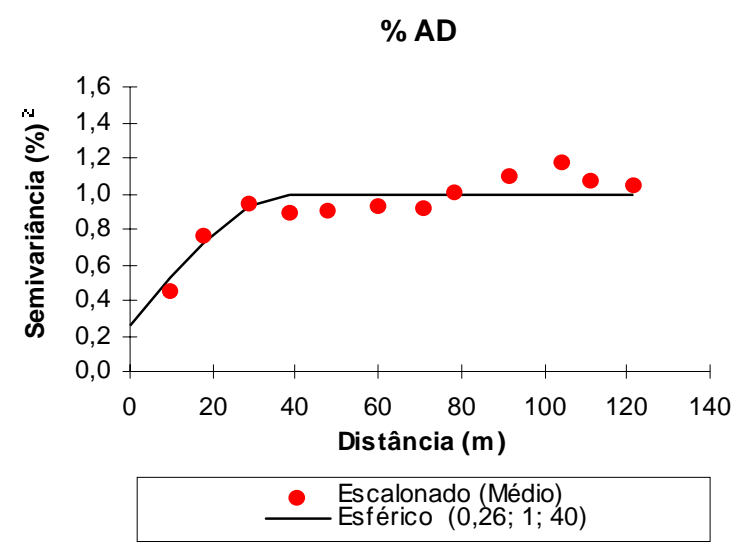

Figura 3. Semivariograma escalonado para semivariâncias dos valores médios de \% água disponível

$\mathrm{O}$ ajuste do modelo esférico com alcance de dependência espacial de $40 \mathrm{~m}$ e efeito pepita de 0,26 (Figura 3 ) possibilitou a construção do mapa de isolinhas representativo das duas profundidades (Figura 4). Nesse mapa, as linhas estão espaçadas de 0,5 unidades de $\mathrm{AD}$ e as linhas mais próximas indicam regiões de maior variabilidade. No lado esquerdo da área, apesar da existência de uma concentração de maiores valores de AD, indicadas pela tonalidade mais escuras da escala de cores, a hipótese da estacionaridade foi assumida na construção dos semivariogramas com base nas análises gráficas e observações feitas por Sousa (1998). Com base na visualização espacial da $\mathrm{AD}$ (Figura 4) dividiu-se a área em quatro sub-áreas de variabilidade semelhante para fins de manejo da irrigação, designadas pelas letras A, B, C e D, de tal forma que as subáreas contivessem valores abaixo, em torno e acima da média (10,28\% e $10,81 \%$ para $0-0,20 \mathrm{~m} 0,20-0,40 \mathrm{~m}$, respectivamente). A letra A (elevação) representa a categoria onde os valores de $\mathrm{AD}$ estão acima de $12 \%$, enquanto as letras B e D (elevações) designam categorias onde os valores estão acima de $9,5 \%$ e abaixo de $12 \%$. A categoria $\mathrm{C}$ constitui uma depressão com

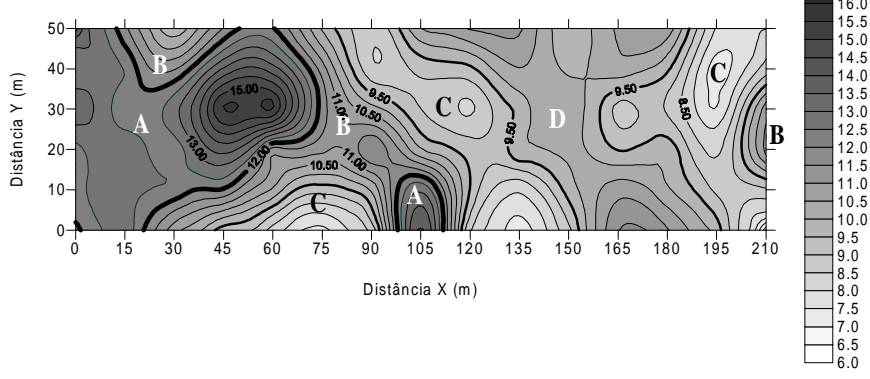

Figura 4. Mapa de isolinhas para valores médios de porcentagem de $\mathrm{AD}$ até $0,40 \mathrm{~m}$ de profundidade 
valor entre $6 \%$ e $9,5 \%$. As categorias B e D são semelhantes quanto a faixa de valores que abrangem, porém em B as linhas estão mais próximas indicando maior variabilidade da AD. Os tempos adequados de precipitação (aspersão) ou de oportunidade (métodos superficiais) deverão ser definidos conforme a capacidade de retenção de água de cada sub-área, e para isso cada uma poderá ter um medidor de umidade (tensiômetros, medidor eletrométrico ou outros mais adequados).

A proposta de manejo na área vai depender do objetivo. Para fins de pesquisa, as sub-áreas podem se constituir em blocos onde os tratamentos podem ser aplicados com maior controle e evitando, dessa forma, o efeito da variabilidade espacial. Do ponto de vista da agricultura, o manejo adequado da irrigação torna-se mais difícil, mas pode ser realizado levando em conta a variabilidade espacial de modo a permitir uma maior eficiência na aplicação de água nas sub-áreas. Pode-se, por exemplo, agrupar áreas com padrões semelhantes de variabilidade e irrigar com base na leitura do tensiômetro da área que possuir valores médios de AD.

\section{CONCLUSÕES}

1. Todas as variáveis estudadas apresentaram estrutura de dependência espacial, sendo porcentagem de silte a que apresentou o menor e o maior alcances de dependência espacial (25 e $60 \mathrm{~m}$ para 0 - 0,20 e 0,20 - 0,40 m, respectivamente).

2. Na profundidade de $0-0,20 \mathrm{~m}$ predominou dependência espacial moderada e na camada de 0,20 - 0,40 cm, uma forte dependência espacial.

3. A variável AD apresentou estrutura de variabilidade semelhante para as duas profundidades amostradas, o que permitiu se construir um semivariograma único com a média dos dados, resultando um alcance de dependência espacial de $40 \mathrm{~m}$.

4. O mapa de isolinhas permitiu a definição de sub-áreas onde o manejo da irrigação pode ser orientado de conformidade com o grau de variabilidade de retenção de água. O manejo poderá ser viabilizado por meio da administração do tempo de precipitação (aspersão) ou do tempo de oportunidade (métodos superficiais).

\section{REFERÊNCIAS BIBLIOGRÁFICAS}

BECKETT, P.H.T.; WEBSTER, R. Soil variability: a review. Soils Fertilizers. Oxford. n.34, p.1-15, 1971.

CAMBARDELLA, C.A.; MOORMAN, T.B.; NOVAK, J.M.; PARKIN, T.B.; KARLEM, D.L.; TURCO, R.F.; KONOPA, A.E. Field scale variability of soil properties in central Iowa soil. Soil Science Society of America Journal, Madison, v.47, p.1501-1511, 1994.
EMBRAPA. Centro Nacional de Pesquisa de Solo (Rio de Janeiro, RJ) Manual de métodos de análise de solo. 2 ed. rev. Rio de Janeiro, 1997. 212p.

GOVERNO DO ESTADO DA PARAÍBA - Superintendência de Obras do Plano de Desenvolvimento do Estado. Projeto de irrigação Capoeira: Estudo de viabilidade (Tomo I - Diagnóstico), 1989. 59p.

GUERRA, P.A.G. Geoestatística operacional, Brasília: Ministério das Minas e Energia - Departamento de Produção Mineral, 1988. $145 p$

GUIMARÃES, E.C. Variabilidade espacial da umidade e da densidade do solo em um Latossolo Roxo. Campinas: ESALQ/ USP, 1993. 138p. Dissertação Mestrado.

KIEHL, E.J. Manual de edafologia e relações solo-planta. São Paulo: Agronômica Ceres, 1979. 220 p.

MORAES, S.O.; LIBARDI. P.L. Problemas metodológicos na obtenção da curva de retenção da água pelo solo. Scientia Agricola, Piracicaba, v.50, n.3, p.383-392, 1993.

OLIVEIRA, J.B. de. Princípios básicos para classificação e sinopse dos principais grandes grupos de solos do mundo. In: Muniz, A.C., ed. Elementos de pedologia. São Paulo: Editora Universidade de São Paulo/Polígono, 1972. p.351-362.

REICHARDT, K.; VIEIRA, S.R.; LIBARDI, P.L. Variabilidade espacial de solos e experimentação de campo. Revista Brasileira Ciência do Solo, Campinas, v.10, n.1, p.1-6, 1986.

SALVIANO, A.A.C. Variabilidade de atributos de solo e de Crotalária juncea em solo degradado do Município de Piracicaba, SP. Piracicaba: ESALQ/USP, 1996. 91 p. Tese Doutorado.

SOUSA, J R. Variabilidade espacial de características fisico-hídricas e de água disponível em um solo aluvial no semi-árido paraibano. Campina Grande: UFPB/CCT, 1998. 65p. Dissertação Mestrado.

SPIEGEL, M.R. Estatística. 2. ed. São Paulo: McGraw-Hill, 1985. $454 p$.

SUDENE - Levantamento exploratório - Reconhecimento de solos do_Estado da Paraíba. Rio de Janeiro, RJ: 1972. 683p. Boletim Técnico n.15.

TRANGMAR, B.B.; YOST, R.S.; UERARA, G. Application of geostatistics to spatial studies or soil properties. Advances in Agronomy, San Diego. v.38, p.45-94, 1985.

VIEIRA, L.S.; SANTOS, P.C.T.C. dos; VIEIRA, M. de N.F. Solos, propriedades, classificação e manejo. Brasília: MEC/ ABEAS, 1988. 154p.

VIEIRA, S.R.; TILLOTSON, P.M.; BIGGAR, J.W.; NIELSEN, D.R. Scaling of semivariograms and kriging estimation of field-measured properties. Revista Brasileira de Ciência do Solo, Viçosa. v.21, p.525-533, 1997.

WARRICK, A.W.; NIELSEN, D.R. Spatial variability of soil physical properties in the field. In: Applications of soil physics. New York: Academic Press, 385p. 1980. 